\title{
A Novel Utility for an Old Method: The Leukocyte Adherence Inhibition Test Is an Easy Way to Detect the Immunoreactive Interference of the Collection Tube Anticoagulant on Cellular Immunoassays
}

\author{
Celso Eduardo Olivier, ${ }^{1}$ Raquel Acácia Pereira Gonçalves dos Santos, ${ }^{2}$ \\ Regiane Patussi Santos Lima, ${ }^{3}$ Daiana Guedes Pinto Argentão, ${ }^{1}$ \\ Grayce Katlen Moreno da Silva, ${ }^{4}$ and Mariana Dias da Silva ${ }^{4}$ \\ ${ }^{1}$ Instituto Alergoimuno de Americana, Rua Chile, 689 Bairro Cechino, 13465-740 Americana, São Paulo, Brazil \\ ${ }^{2}$ Faculty of Nursing, Anhanguera University of Santa Bárbara, São Paulo, Brazil \\ ${ }^{3}$ Faculty of Biomedicine, Anhanguera University of Santa Bárbara, São Paulo, Brazil \\ ${ }^{4}$ Faculty of Pharmacy, Methodist University of Piracicaba, São Paulo, Brazil
}

Correspondence should be addressed to Celso Eduardo Olivier; docsystems@docsystems.med.br

Received 7 December 2013; Revised 24 December 2013; Accepted 9 January 2014; Published 18 February 2014

Academic Editor: Seetharama D. Satyanarayanajois

Copyright (C) 2014 Celso Eduardo Olivier et al. This is an open access article distributed under the Creative Commons Attribution License, which permits unrestricted use, distribution, and reproduction in any medium, provided the original work is properly cited.

\begin{abstract}
The leukocyte adherence inhibition (LAI) test was designed to evaluate the presence of cellular immunoreactivity against specific antigens and is highly sensitive to analytical interference. To compare the performance of the LAI test among plasmas obtained with different anticoagulants, 36 blood samples of allergic subjects were divided into three tubes with heparin, sodium citrate, and EDTA. The adherence was assessed with the hemocytometer technique. The mean adherence of each group was compared as well as the number of tests with inappropriate adherence $(<25 \%)$. Of the 36 tests, there were 4,9 , and 21 with inappropriate adherence in the groups of heparin, citrate, and EDTA, respectively. Of these last, 5 samples also did not demonstrate proper adherence with sodium citrate and 1 sample did not show proper adherence with any anticoagulant. The anticoagulant in the collection tube can elicit a specific immune response, becoming an interferent for the LAI test. In this case, there is the possibility of redoing the test using another anticoagulant. Performed with different anticoagulants, the LAI test may be used as a preanalytical tool for choosing the less immunoreactive anticoagulant for more complex immunoassays.
\end{abstract}

\section{Introduction}

The evaluation of the immune status by the leukocyte adherence inhibition (LAI) test may be achieved by three main distinct methods: the method of the hemocytometer [1], the method of the tube [2], and the method of the microplate $[3,4]$. The original hemocytometer LAI test described for detection of cell-mediated tumour immunity and serum blocking factors [5] was initially used to assess cancer immunoreactivity [6-8] and further also used to describe antigenspecific cell-mediated responses to Candida, house dust, PPD, and chronic dermatophytic infections $[9,10]$. The allergen-specific LAI test carried by the tube method was designed to monitor cell responses to ex vivo allergen challenges and was able to differentiate the plasma of subjects allergic to Dermatophagoides farinae from the plasma of subjects allergic to Artemisia vulgaris and from the plasma of nonallergic subjects [11]. Failure of leukocytes to adhere to glass after ex vivo challenge with specific antigens depends mostly on cellular mechanisms resulting from the interactions of B cells, $T$ cells, monocytes and macrophages $[12,13]$, and several humoral factors, among them cytokines and antibodies [14]. Several mechanisms have been implied as responsible for the production of this phenomenon that seems to be just the final 


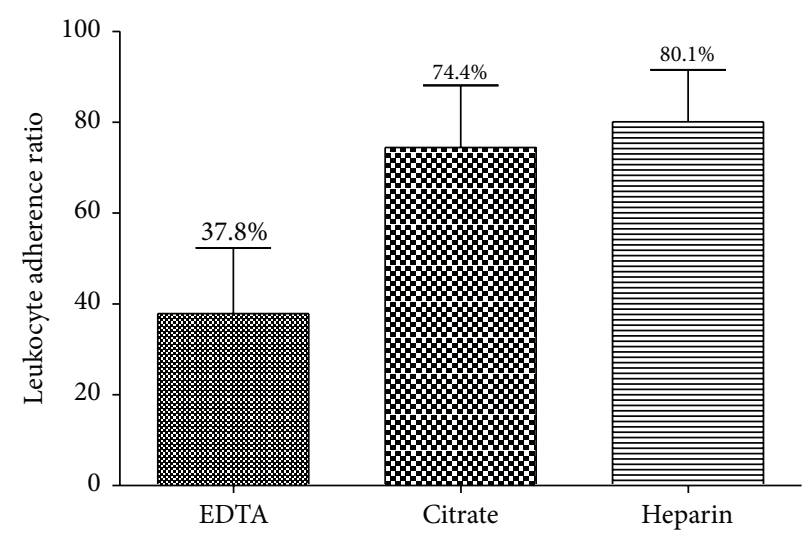

FIGURE 1: Mean leukocyte adherence ratio (LAR) of the unchallenged plasma of 36 subjects used as controls for the leukocyte adherence inhibition test performed with EDTA, sodium citrate, or heparin.

indicator of leukocyte activation or immunoreactivity [15]. When activated in the presence of a specific antigen, leukocytes interact with each other by releasing soluble factors that interfere with glass adherence, in contrast to what is observed at the unchallenged control plasma [16]. The antigen-specific inhibition of the leukocyte adherence to the glass was described to be dependent on leukotrienes [17], interferon [18], platelet-derived factors [19], T-cell lymphokines [20], and Fröhlich electromagnetic radiation [21]. Each one of the three main methods (and its variants) may be based on distinct immune mechanisms [22] that result in the same final phenomenon, turning the results even more complex to interpret [23]. However, whatever the technique used, the LAI test depends on the adherence control, that is, the baseline adherence of the leukocytes to glass without any antigen challenge. Ideally, the nonadherence index (NAI) of the unchallenged plasma must be equal to zero [11]. The LAI test has been described by a great variety of techniques with diverse levels of elaboration, equipment, and reagents that turned it into a not so convenient assay to be held into a routine analysis of a clinical laboratory [16, 24]. Besides increasing the assay's complexity, the addition of reagents and unnecessary steps introduces some immunoreactive analytical interferents that compromise the reproducibility and the credibility of the assay $[25,26]$. To evaluate specifically the interference of the tube collection anticoagulant on the leukocyte adherence to the vitro, we studied the plasma obtained with three different anticoagulants of 36 allergic subjects submitted simultaneously to three hemocytometer adherence tests.

\section{Materials and Methods}

2.1. Study Design and Subjects. This descriptive study was conducted over a period of six months with the fresh plasma of 36 food-allergic human subjects in a previous preanalytical study to assess the differences of the LAI test challenged with native and polymerized beta-lactoglobulin [27]. To evaluate the interference of tube collection anticoagulant over the natural leukocyte adherence to the glass, we performed three paired hemocytometer adherence tests with plasma obtained with heparin, sodium citrate, and EDTA. This study was submitted to and approved by the Institutional Research Ethics Board and was registered in the Brazilian National Ethics Research System (SISNEP 409/2008). In accordance with the Helsinki Declaration, signed consent forms were obtained from all subjects. Group comparisons were performed with the paired $t$-test. The data are reported as the arithmetic mean with 95\% confidence intervals (CI). Statistical analyses were performed with GraphPad Prism for Windows (version 5.0; GraphPad Software, Inc., San Diego, CA, USA).

2.2. Hemocytometer Leukocyte Adherence Assessment. After blood collection, the sample was separated into three plastic collection tubes with heparin, sodium citrate, and EDTA. After blood spontaneous sedimentation $\left(60\right.$ minutes at $\left.37^{\circ} \mathrm{C}\right)$, the peripheral blood leukocyte-rich plasma fraction was collected from each tube with a plastic Pasteur pipette and allocated in standard Neubauer hemocytometer chambers with plain glass surface and left for 2 hours at $37^{\circ} \mathrm{C}$ in a humidified atmosphere to allow leukocyte adherence. After the leukocyte counting, the hemocytometer chamber was immersed into a beaker with $\mathrm{PBS}$ at $37^{\circ} \mathrm{C}$ and the coverslip was removed and replaced by a clean one. The remaining cells were counted on the same hemocytometer squares as examined previously. The leukocyte adherence ratio (LAR) was calculated as the percentage of the number of leukocytes remaining after immersion $(\times 100)$ divided by the number of leukocytes counted before immersion (\%). The nonadherence index (NAI) was calculated by subtracting this result from 100 (\%).

\section{Results}

3.1. The Mean Leukocyte Adherence Ratio. The mean leukocyte adherence ratio (LAR) of the tests performed with EDTA was $37.8 \%$ (CI 23.4 to 52.3 ). The mean LAR of the tests performed with sodium citrate was $74.4 \%$ (CI 60.7 to $88.1 \%$ ). The mean LAR of the tests performed with heparin was $80.1 \%$ (CI 68.7 to 91.5 ) (see Figure 1). There was no significant difference in the paired $t$-test of the mean of the differences between the sodium citrate LAR and heparin LAR (5.7\%; CI 8.0 to 
19.4; $P=0.4$ ). The mean of the differences in the paired $t$-test between EDTA LAR and sodium citrate LAR was significant $(36.5 \%$; CI $16 \%$ to $57 \% ; P=0.0009)$. The mean of the differences in the paired $t$-test between EDTA LAR and heparin LAR was significant (42.2\%; CI 23.3\% to 61.1\%; $P<$ $0.0001)$.

3.2. The Categorical Nonadherence Index. In order to categorically classify the results, we arbitrarily establish two categories: (A) tests with an NAI $>75 \%$ (or LAR $\leq 25 \%$ ) were classified as improper to act as a control for the LAI assay and (B) tests with NAI $\leq 75 \%$ (or LAR > 25\%) were considered proper to act as a control for the LAI assay. There were 4 tests considered improper to act as a control in the heparin group. There were 9 tests considered improper to act as a control in the sodium citrate group. Among the 21 subjects who did not demonstrate proper NAI when the blood was collected with EDTA, 5 subjects also did not demonstrate proper NAI to sodium citrate and 1 subject did not demonstrate proper NAI to any anticoagulant (see Table 1).

\section{Discussion}

Immunobiology is beginning an era where the ex vivo assays are intending to replace the in vivo tests to evaluate immunoreactivity against specific antigens (allergen challenges) [28]. The diagnosis of specific immunoreactivity is a crescent need in several clinical fields, such as the rheumatology, the neurology, the endocrinology, the infectology, the allergology, and the immunology. When there is a need to evaluate the humoral immunoreactivity, there are hundreds of readily available resources to offer help to the physicians; however, the ex vivo assessment of the cellular immunoreactivity is yet an unexplored field. The LAI test is an ex vivo test designed to evaluate the specific cellular/humoral immune response against specific antigens. Unfortunately, immunoassays are imperfect [29]. The researchers who have used the LAI test have published some amazing results, but the protocol standardization of this immunoassay for implementation in clinical analytical practice has been hampered by the excessive number of different methods and analytical interferents that turned this assay complex and time consuming. In this work, we minimized the steps and excluded unnecessary reagents to make a simpler assay and define its main analytical interference that may be the anticoagulant from the collection tube. The LAI test is antigen-specific but it is not mechanism-specific. Since there are several mechanisms that can produce the glass nonadherence phenomenon, this common final result represents, a priori, just an unspecific marker of immunoreactivity against the specific antigen used for the challenge. In this work, we did not execute the entire LAI test procedure. The LAI test has 2 steps. The first step is the control of the procedure (control step). The second step is the evaluation of the inhibition of adherence produced by the antigen ex vivo challenge (challenge step). In the control step the analyst will check the natural adherence of the leukocytes to validate the challenge step. If the control step, does not present a proper adherence, the adherence step is not validated. The control
TABLE 1: Leukocyte adherence ratio (LAR) of the 36 subjects' unchallenged plasmas submitted to the hemocytometer leukocyte adherence assay.

\begin{tabular}{|c|c|c|c|}
\hline Subject & EDTA LAR (\%) & Citrate LAR (\%) & Heparin LAR (\%) \\
\hline 1 & 2 & 6 & 84 \\
\hline 2 & 100 & 0 & 24 \\
\hline 3 & 0 & 16 & 55 \\
\hline 4 & 92 & 94 & 95 \\
\hline 5 & 0 & 100 & 100 \\
\hline 6 & 0 & 98 & 100 \\
\hline 7 & 50 & 84 & 90 \\
\hline 8 & 91 & 100 & 11 \\
\hline 9 & 89 & 98 & 100 \\
\hline 10 & 18 & 100 & 100 \\
\hline 11 & 8 & 100 & 100 \\
\hline 12 & 2 & 92 & 32 \\
\hline 13 & 88 & 8 & 100 \\
\hline 14 & 91 & 91 & 100 \\
\hline 15 & 71 & 97 & 100 \\
\hline 16 & 0 & 100 & 50 \\
\hline 17 & 0 & 100 & 100 \\
\hline 18 & 0 & 98 & 100 \\
\hline 19 & 98 & 99 & 96 \\
\hline 20 & 82 & 93 & 95 \\
\hline 21 & 100 & 0 & 0 \\
\hline 22 & 0 & 100 & 100 \\
\hline 23 & 95 & 96 & 95 \\
\hline 24 & 97 & 100 & 100 \\
\hline 25 & 0 & 98 & 100 \\
\hline 26 & 8 & 0 & 12 \\
\hline 27 & 100 & 100 & 100 \\
\hline 28 & 2 & 96 & 100 \\
\hline 29 & 35 & 0 & 100 \\
\hline 30 & 0 & 100 & 0 \\
\hline 31 & 21 & 98 & 100 \\
\hline 32 & 0 & 99 & 100 \\
\hline 33 & 20 & 0 & 45 \\
\hline 34 & 3 & 100 & 100 \\
\hline 35 & 0 & 23 & 100 \\
\hline 36 & 0 & 95 & 100 \\
\hline
\end{tabular}

step also serves to calculate the adherence inhibition. The formula to calculate the adherence inhibition is a ratio between the adherence of the challenge step and the adherence of the control step. The LAI test may be influenced by many interferents. The nature of the interferences is not determined yet, but there is a possibility that it may be immune and specific. In this work, we were not studying adherence to glass, but interferents for the LAI test, which is a basic tool to study cellular adherence. The objective was to standardize the test. The initial proposition of this work was to determine if specific immunoreactivity against heparin (a highly sulfated glycosaminoglycan) was a factor able to produce interference with the test. There are several reports 
of IgE-mediated and non-IgE-mediated allergy to heparin $[30,31]$, but, unexpectedly for us, heparin presented the better profile to perform the test, and even, among the few subjects where heparin produced interference, we were not able to prove sensitization by skin prick tests. The utilization of an allergic population was taken to increase the probability of random immunoreactivity against the anticoagulant and for the fact that one of our next steps is to study the LAI test to detect immunoreactivity against relevant allergens in allergic subjects. The primary objective of this previous study was to determine which anticoagulant was better for the LAI test in an immunoreactive population. The results were unexpected for us and highly reproducible. The crucial point of the LAI test is the washing step. If it is not performed properly, the cells can be ripped of the glass when the coverslip is replaced. However, for a well-trained and experienced clinical analyst, the washing step is very simple and highly reproducible (see Video 1 in Supplementary Material available online at http://dx.doi.org/10.1155/2014/860427). But the main point to assure us that the technical procedure was performed properly is the double chamber hemocytometer covered by the same coverslip. The Neubauer device has 2 chambers that were filled with the plasma of the same subject isolated with two different anticoagulants. So, the washing procedure was just the same in the 2 chambers and we observed clearly $100 \%$ of adherence in one of the chambers and $0 \%$ of adherence in the other chamber. The serum was of the same subject and was collected at the same time; there was no antigen challenge, and, therefore, the only variable was the anticoagulant. We did not perform the tests in duplicate for all subjects, but, in the subjects on whom we repeated the test in another occasion (for the antigen challenge), the result was similar. In the particular assay described in this paper, we did not use any antigen challenge, since the objective was to study the influence of the collection tube anticoagulant over the leukocyte adherence to the glass, so the antigen evaluated was the very anticoagulant used to obtain the plasma sample. Therefore, the failure to adhere to the glass suggests a specific plasmatic immunoreactivity to the very anticoagulant. All of the three anticoagulants showed this possibility to some subjects, and all of the three anticoagulants did not react at all with some plasma samples, denoting an NAI = zero. Heparin showed the more convenient profile and EDTA the less convenient profile to perform LAI test. This implies that if a particular anticoagulant does not show a proper adherence to act as the control, the test may be repeated with the use of another one. The high prevalence of immunoreactivity against the anticoagulants in this particular series may be derived from the choice of allergic subjects to perform the tests, since we were randomly searching for immunoreactivity against the anticoagulants. Possibly, in healthy people, one must find a lower frequency of specific immunoreactivity against the collection tube anticoagulant. As well as the anticoagulants, other biological products added to the test, due to its high protein content (such as fetal calf serum), or because of repetitive motifs (such as dextran), were also potentially immunogenic and may act as analytical interferents to the LAI test. Several authors included in the first step of the LAI test the enrichment of the leukocyte-rich plasma fraction by use of dextran [32,33]. Dextran is a complex branched bacterial polysaccharide obtained from Leuconostoc mesenteroides. We used to add it to the initial blood sedimentation step until we found a patient who reacted with it, promoting a total inhibition of the adherence. The reexecution of the LAI test without dextran restored the normal adherence to conclude the test. Since then, we have not used dextran anymore, likewise several other researches [10, 11]. The analytical interference of dextran was already a reason for discrediting the LAI test among some researches [26]. Similarly, we do not use enrichment solutions, such as fetal calf serum or Medium 199, neither add $\mathrm{CO}_{2}$ to the air. Besides the possibility to act as analytical interferents, these elements are not essential to the test, turning the assay more expensive and complex. Like all tests performed ex vivo, the LAI test must be done with care to keep leukocytes vitality. Therefore, the whole procedure must be performed at $37^{\circ} \mathrm{C}$ and must follow the recommended times. The period of two hours for adhesion to glass time was shown to be ideal, because when we left them for an hour or one hour and a half, the leukocytes did not show satisfactory adherence. If left for more than two hours, in addition, the leukocytes initiate the process of cell death and loss of adherence. To keep the vitality of leukocytes, we do not recommend fasting for blood collection. The presence of nutrients in the plasma seems to preserve the vitality of the cells for a longer period. The particular sensitivity of the LAI test to analytical interferents may be converted into a tool to evaluate analytical interference of biological reagents in similar immunoassays. Due to its simplicity, the LAI test may be useful as a preanalytical tool to investigate the presence of immunoreactivity against specific reagents and/or anticoagulants as a triage test to prevent analytical interference and choose the more convenient anticoagulant to obtain the plasma sample. This preanalytical triage may be particularly helpful, before the employ of more complex, time-consuming, and expensive assays as the basophil challenge test [34], for instance, or any immunoassay where the plasma is obtained with the help of anticoagulants to study the cellular secretion of cytokines, chemokines, and/or soluble receptors after specific stimulation or antigen challenge [35].

\section{Conclusion}

We concluded that the anticoagulant in the collection tube may be the target of a specific host immunological reaction, becoming an analytical interferent for the LAI test, in special, and for cellular immunoassays, in general. Due to its particular sensitivity to analytical interference, the LAI test may be used as a preanalytical tool to help in the decision to choose the most convenient anticoagulant for each subject in clinical or research analysis where specific immunoreactivity remains a possibility.

\section{Abbreviations}

LAI test: Leukocyte adherence inhibition test

LAR: Leukocyte adherence ratio

NAI: Nonadherence index. 


\section{Conflict of Interests}

The authors have declared that they have no conflict of interests.

\section{Acknowledgment}

The present study was supported by the Instituto Alergoimuno de Americana.

\section{References}

[1] A. E. Maluish and W. J. Halliday, "Hemocytometer leukocyte adherence inhibition technique," Cancer Research, vol. 39, no. 2, pp. 625-626, 1979.

[2] D. M. P. Thomson, "Demonstration of tube leukocyte adherence inhibition assay with coded samples of blood," Cancer Research, vol. 39, no. 2, pp. 627-629, 1979.

[3] P. G. Holt, P. J. Fimmel, L. M. Finlay-Jones, and R. L. Flower, "Evaluation of the microplate leukocyte adherence inhibition test and its reproducibility, sensitivity, and relationship to other tests of cellular immunity," Cancer Research, vol. 39, no. 2, pp. 564-569, 1979.

[4] G. F. Santini, S. Battistin, and P. de Paoli, "A method for the determination of the adherence of granulocytes to microtitre plates," Journal of Immunological Methods, vol. 100, no. 1-2, pp. 153-156, 1987.

[5] W. J. Halliday and S. Miller, "Leukocyte adherence inhibition: a simple test for cell-mediated tumour immunity and serum blocking factors," International Journal of Cancer, vol. 9, no. 3, pp. 477-483, 1972.

[6] Y. Berner, A. Fink, and A. Shani, "Diagnostic value of the computerized tube leukocyte adherence inhibition (LAI) assay for human colorectal, breast and lung cancers," Oncology, vol. 43, no. 5, pp. 327-334, 1986.

[7] R. Dgani, A. Shani, U. Elchalal, N. Harpaz, Z. Bentwich, and A. Fink, "The leukocyte adherence inhibition test (LAI) in preoperative diagnosis of epithelial ovarian cancer," Gynecologic Oncology, vol. 49, no. 3, pp. 349-353, 1993.

[8] V. Holan, O. Sibl, and M. Hasek, "Monitoring of antitumor immunity in patients with larynx cancer by tube leukocyte adherence inhibition assay," Cancer Research, vol. 39, no. 2, pp. 651-653, 1979.

[9] B. A. J. Walters, J. E. D. Chick, and W. J. Halliday, "Cell mediated immunity and serum blocking factors in patients with chronic dermatophytic infections," International Archives of Allergy and Applied Immunology, vol. 46, no. 6, pp. 849-857, 1974.

[10] T. Kuratsuji, "Studies on leukocyte adherence inhibition test. Part II. Clinical application of LAI test to detect delayed type hypersensitivity in infants and children," Keio Journal of Medicine, vol. 30, no. 2, pp. 65-69, 1981.

[11] A. Fink, L. Heller, A. Eliraz et al., "Allergen-specific leukocyte adherence inhibition (LAI) assay: sensitivity, specificity and mechanism," Immunology Letters, vol. 16, no. 1, pp. 65-70, 1987.

[12] I. S. Dunn and W. J. Halliday, "Subpopulations of splenic T and B lymphocytes producing and regulating leukocyte adherence inhibition factor," Cellular Immunology, vol. 56, no. 2, pp. 465477, 1980.

[13] I. S. Dunn and W. J. Halliday, "Interactions between T and B lymphocytes and macrophages in the production of leukocyte adherence inhibition factor," Cellular Immunology, vol. 52, no. 1, pp. 48-61, 1980.

[14] M. H. Goldrosen, A. J. Russo, and J. H. Howell, "Cellular and humoral factors involved in the mechanism of the microleukocyte adherence inhibition reaction," Cancer Research, vol. 39, no. 2, pp. 587-592, 1979.

[15] T. Appelboom, N. Dourov, R. Gortz, and J. Wybran, "Leukocyte adherence inhibition test: cellular requirements for the elaboration of a polymorph adherence inhibition activity," Clinical Immunology and Immunopathology, vol. 20, no. 1, pp. 63-73, 1981.

[16] W. J. Halliday, "Historical background and aspects of the mechanism of leukocyte adherence inhibition," Cancer Research, vol. 39, no. 2, pp. 558-563, 1979.

[17] A. Fink, H. Bibi, and A. Eliraz, "Leukotrienes (LTC4, LTD4) confer glass non-adherence on leukocytes of asthmatic individuals. Dependency on cycloxygenase products and calcium ion," Immunology Letters, vol. 10, no. 6, pp. 319-323, 1985.

[18] A. Fink, R. Shahin, and A. Eliraz, "Interferon modulates the leukotriene C4-induced non-adherence properties of leukocytes: acquisition of an asthmatic phenotype," Immunology Letters, vol. 10, no. 3-4, pp. 159-163, 1985.

[19] I. Kazuhisa and Y. Tatsuhisa, "Platelet-derived neutrophil adherence-inhibiting factor in humans," Blood, vol. 76, no. 11, pp. 2368-2373, 1990.

[20] A. E. Powell, A. M. Sloss, and R. N. Smith, "Leukocyteadherence inhibition: a specific assay of cell-mediated immunity dependent on lymphokine-mediated collaboration between T lymphocytes," Journal of Immunology, vol. 120, no. 6, pp. 1957-1966, 1978.

[21] J. Pokorny, A. Jandova, and J. Kobilkova, "Frohlich electromagnetic radiation from human leukocytes: implications for leukocyte adherence inhibition test," Journal of Theoretical Biology, vol. 102, no. 2, pp. 295-305, 1983.

[22] R. Schimke, V. Holan, and O. Sibl, "Comparison of the hemocytometer and tube modifications of the leukocyte adherence inhibition assay II. Application of the both modifications for detection of anti-tumor immunity in man," Neoplasma, vol. 28, no. 3, pp. 257-264, 1981.

[23] D. M. P. Thomson, Assessment of Immune Status by the Leukocyte Adherence Inhibition Test, Academic Press, New York, NY, USA, 1982.

[24] R. L. Nutter and J. D. Kettering, "Leukocyte adherence inhibition: history, biological basis and clinical usefulness," European Journal of Cancer and Clinical Oncology, vol. 23, no. 9, pp. 12571260, 1987.

[25] W. Stepniewicz, H. Tchórzewski, and M. Luciak, "Factors influencing human leukocyte adherence in vitro," Folia Haematologica, vol. 110, no. 3, pp. 373-379, 1983.

[26] H. Tchórzewski, M. Luciak, and W. Stepniewicz, "Factors influencing human leukocyte adherence in vivo," Folia Haematologica, vol. 110, no. 3, pp. 380-386, 1983.

[27] C. E. Olivier, R. P. Lima, D. G. Pinto et al., "In search of a tolerance-induction strategy for cow's milk allergies: significant reduction of beta-lactoglobulin allergenicity via transglutaminase/cysteine polymerization," Clinics, vol. 67, no. 10, pp. 11711179, 2012.

[28] A. Singh, S. Holvoet, M. Weiss, M. Beaumont, A. W. Zuercher, and A. Mercenier, "Increased IL-5 and IL-13 cytokine level in ex vivo stimulated whole blood cells from grass pollen allergic donors correlate with seasonal exposure," Results in Immunology, vol. 1, no. 1, pp. 18-23, 2011. 
[29] A. N. Hoofnagle and M. H. Wener, "The fundamental flaws of immunoassays and potential solutions using tandem mass spectrometry," Journal of Immunological Methods, vol. 347, no. 1-2, pp. 3-11, 2009.

[30] A. Trautmann and C. S. Seitz, "Heparin allergy: delayed-type non-IgE-mediated allergic hypersensitivity to subcutaneous heparin injection," Immunology and Allergy Clinics of North America, vol. 29, no. 3, pp. 469-480, 2009.

[31] N. Hallai, T. M. Hughes, and N. Stone, “Type I and type IV allergy to unfractionated heparin and low-molecular-weight heparin with no reaction to recombinant hirudin," Contact Dermatitis, vol. 51, no. 3, pp. 153-154, 2004.

[32] A. W. Bullen and M. S. Losowsky, "Comparison of a leucocyte adherence test with the leucocyte migration inhibition test and skin reactivity to PPD," Clinical and Experimental Immunology, vol. 31, no. 3, pp. 408-413, 1978.

[33] A. W. Tong, D. R. Burger, and P. Finke, "Assessment of the mechanism of the leukocyte adherence inhibition test," Cancer Research, vol. 39, no. 2, pp. 597-603, 1979.

[34] N. Sousa, R. Martínez-Aranguren, M. Fernández-Benitez, F. Ribeiro, and M. L. Sanz, "Comparison of basophil activation test results in blood preserved in acid citrate dextrose and EDTA," Journal of Investigational Allergology and Clinical Immunology, vol. 20, no. 6, pp. 535-536, 2010.

[35] I. Agalliu, X. Xue, M. Cushman et al., "Detectability and reproducibility of plasma levels of chemokines and soluble receptors," Results in Immunology, vol. 3, pp. 79-84, 2013. 

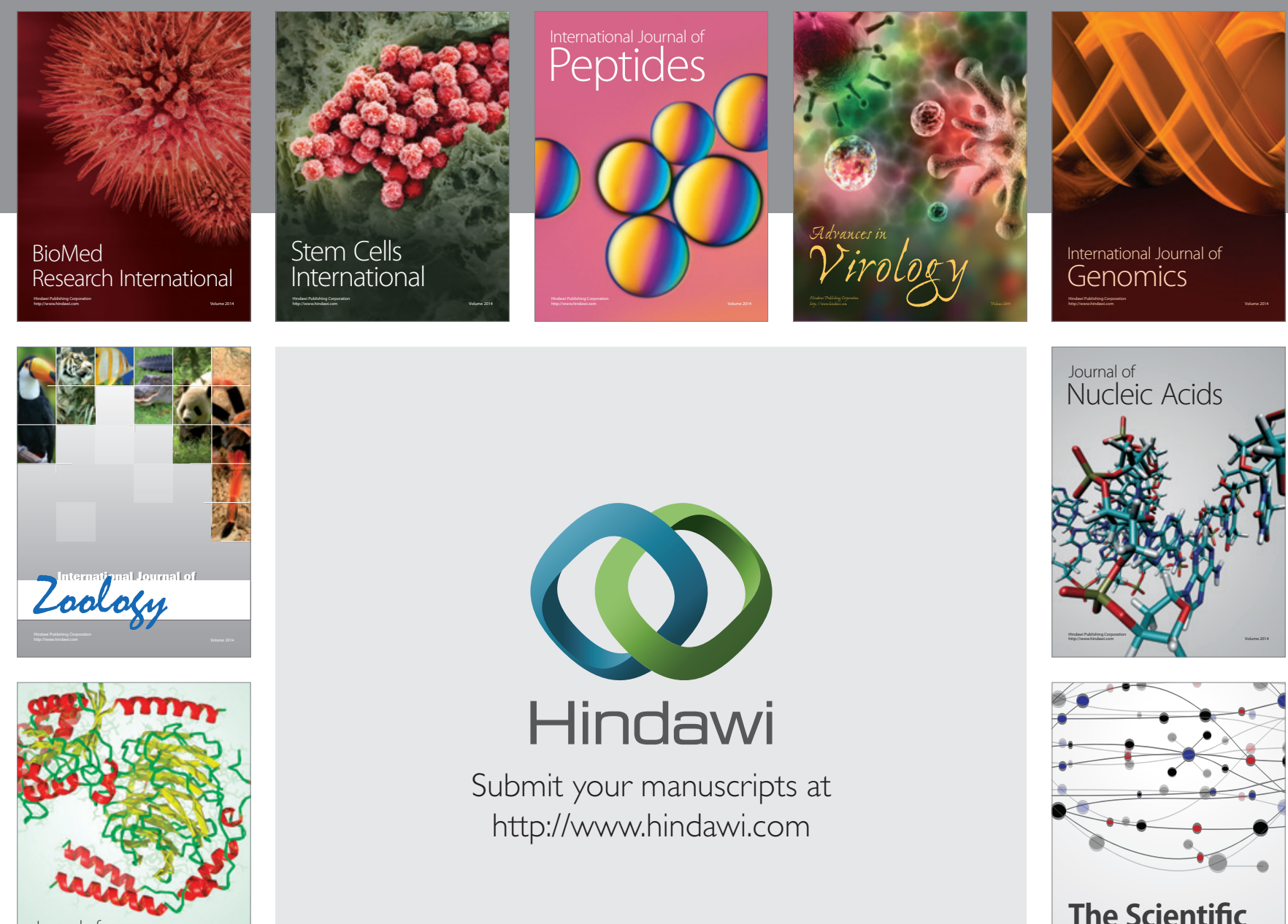

Submit your manuscripts at

http://www.hindawi.com

Journal of
Signal Transduction
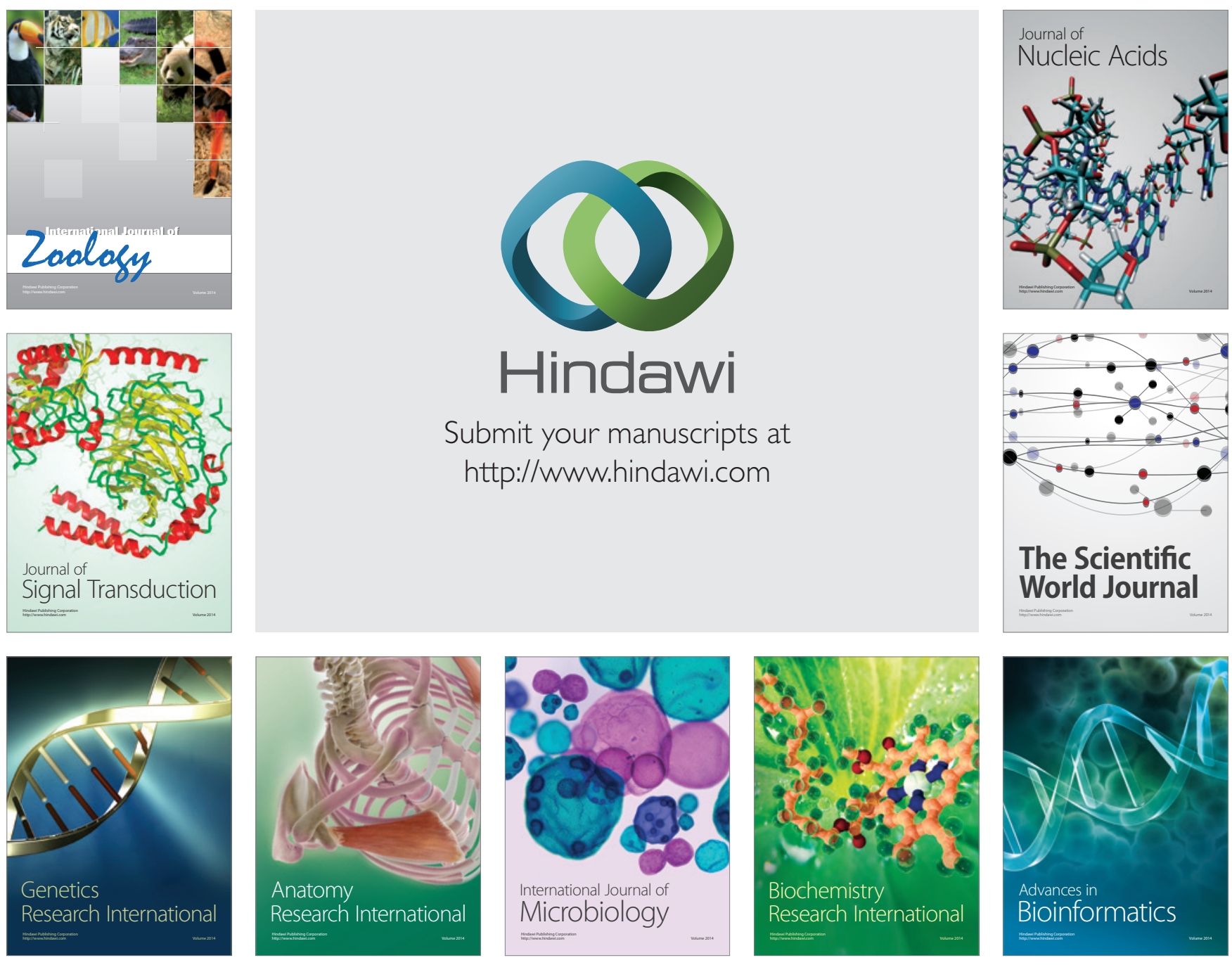

The Scientific World Journal
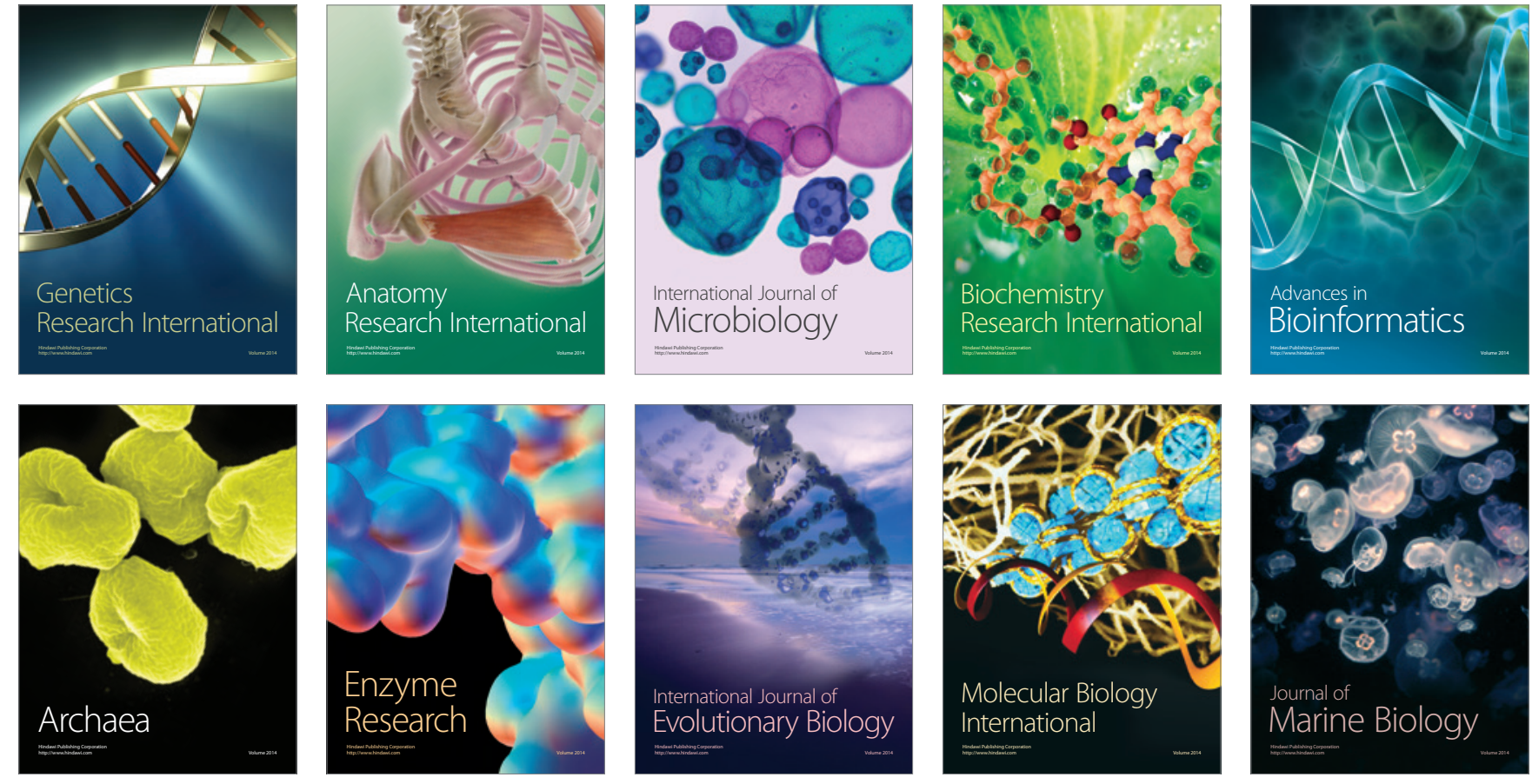